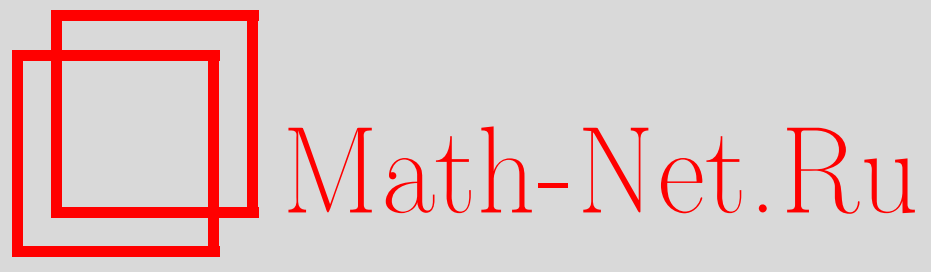

Н. П. Долбилин, А. Н. Магазинов, Локально антиподальные множества Делоне, УМН, 2015, том 70, выпуск $5,179-180$

DOI: https://doi.org/10.4213/rm9685

Использование Общероссийского математического портала Math-Net.Ru подразумевает, что вы прочитали и согласны с пользовательским соглашением http://www . mathnet.ru/rus/agreement

Параметры загрузки:

IP : 54.84 .234 .179

26 апреля 2023 г., 17:07:19

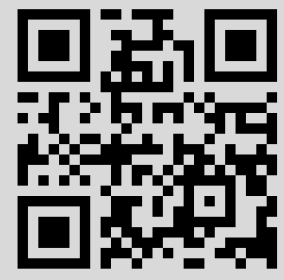




\section{Локально антиподальные множества Делоне}

\section{Н. П. Долбилин, А. Н. Магазинов}

В заметке излагаются новые результаты локальной теории правильных и кристаллических систем, начатой в [2]. Результаты относятся к важному случаю дискретных множеств, для которых группа симметрий каждого $2 R$-кластера содержит центральную симметрию. Основной результат заметки - теорема 3 о единственности множества Делоне с центрально симметричными $2 R$-кластерами при условии, что задан некоторый его $2 R$-кластер. Из теоремы следует ряд доказанных ранее утверждений о "локально антиподальных" множествах, а также теорема 4 о структуре множества Делоне с антиподальными $2 R$-кластерами, из которой, в свою очередь, следует, что локально антиподальное множество Делоне является кристаллической структурой, хотя никаких дополнительных условий на количество классов $2 R$-кластеров в множестве не налагается.

Множество $X \subset \mathbb{R}^{d}$ называют множеством Делоне с параметрами $r$ и $R$, где $r, R>0$ (или $(r, R)$-системой - см., например, [1]), если выполняются два условия:

$1)$ открытый $d$-шар $B_{y}^{\circ}(r)$ радиуса $r$ с центром в произволъной точке $y \in \mathbb{R}^{d}$ содержит не более одной точки из $X$ :

$$
\#\left(B_{y}^{\circ}(r) \cap X\right) \leqslant 1 ;
$$

2 ) замкнутый $d$-шар $B_{y}(R)$ радиуса $R$ содержит хотя бы одну точку из $X$ :

$$
\#\left(B_{y}(R) \cap X\right) \geqslant 1 .
$$

Пусть $X$ - множество Делоне, $x \in X, \rho$ - положительное число, тогда множество $C_{x}(\rho):=X \cap B_{x}(\rho)$ и будем называть $\rho$-кластером точки $x$. Два $\rho$-кластера $C_{x}(\rho)$ и $C_{x^{\prime}}(\rho)$ назовем эквивалентными, если существует движение $g$ такое, что

$$
g: x \mapsto x^{\prime} \quad \text { и } \quad g: C_{x}(\rho) \rightarrow C_{x^{\prime}}(\rho) .
$$

Если для данного множества Делоне $X$ при каждом $\rho>0$ число классов эквивалентных $\rho$-кластеров конечно, то говорят, что $X$ - множество конечного типа. Для множества Делоне $X$ конечного типа обозначим число классов $\rho$-кластеров через $N(\rho)$. Известно, что для данного $X$ конечность числа классов $2 R$-кластеров, $N(2 R)<\infty$, гарантирует конечность числа $N(\rho)$ классов $\rho$-кластеров для любого фиксированного $\rho>0$. Заметим, что $N(\rho)$ есть положительная, целочисленная, неубывающая, непрерывная справа функция. Важными примерами множеств Делоне конечного типа являются понятия правильной системы и кристалла.

Правильная система - это множество Делоне, группа симметрий которого действует транзитивно, т.е. для любой пары точек $x$ и $x^{\prime}$ из $X$ найдется движение $g$ пространства $\mathbb{R}^{d}$ такое, что

$$
g: x \mapsto x^{\prime} \quad \text { и } \quad g: X \rightarrow X .
$$

Нетрудно показать, что правильная система является орбитой точки относительно некоторой кристаллографической группы.

Кристаллом называют множество Делоне, являющееся орбитой конечного множества $X_{0}$ относительно некоторой кристаллографической группы $G: G \cdot X_{0}$.

Множество Делоне конечного типа является правильной системой, если $N(\rho) \equiv 1$ для всех $\rho>0$, и кристаллом, если перечисляющая функция ограничена: $N(\rho) \leqslant$ $m<\infty$. Если $m=1$, то кристалл является правильной системой.

Назовем множество Делоне $X$ локально антиподалъным, если $2 R$-кластер $C_{x}(2 R)$ для каждой точки $x \in X$ центрально симметричен относительно $x$. В [3] были доказаны две теоремы о локально антиподальных множествах.

Исследование выполнено за счет гранта Российского научного фонда (проект № 14-11-00414). DOI: $10.4213 / \mathrm{rm} 9685$ 
Теорема 1 (об антиподальности множества). Локально антиподальное множество Делоне в целом антиподально относительно каждой своей точки.

Теорема 2. Пусть для локально антиподального множества Делоне $X$ выполнено равенство $N(2 R)=1$. Тогда $X$ является правильной системой.

Основной результат заметки - следующая теорема.

Теорема 3 (единственности). Пусть $X$ и $Y$ - локально антиподальные множества Делоне с параметром $R$. Предположим, что $x \in X \cap Y u C_{x}(2 R)=C_{x}^{\prime}(2 R) \subset$ $X \cap Y$. Тогда $X=Y$.

Здесь $C_{y}^{\prime}(2 R), y \in Y$, обозначает $2 R$-кластер в множестве $Y \cap B_{y}(2 R)$. Как утверждает теорема, если $X$ и $Y$ имеют общий $2 R$-кластер и все $2 R$-кластеры в обоих множествах центрально симметричны, то множества совпадают в целом.

Из этой теоремы немедленно следуют доказанные в [3] теоремы 1 и 2. Действительно, по условию теоремы 1 симметрия $\sigma$ относительно точки $x$ переводит $C_{x}(\rho)$ в себя, а $X$ в $Y:=\sigma(X)$. Тогда по теореме 3 справедливо равенство $X=\sigma(X)$, что доказывает теорему 1.

Далее, по условию теоремы 2 для любых двух точек $x, x^{\prime} \in X$ существует движение $g$ такое, что $g(x)=x^{\prime}$ и $g\left(C_{x}(2 R)\right)=C_{x^{\prime}}(2 R)$. Рассмотрим множество $Y:=g(X)$, для него имеем $Y \cap X \supset C_{x^{\prime}}(2 R)$. По теореме 3 получаем, что $X=Y$, т. е. $X=g(X)$, откуда следует теорема 2.

Наиболее интересное следствие теоремы 3 - это приводимая ниже теорема 4 о структуре локально антиподального множества Делоне. Произвольная точечная решетка $\Lambda$ является локально антиподальным множеством. Если добавить к ней параллельные решетки, сдвинутые во все или в некоторые "полуцелые" точки для этой решетки, получатся, очевидно, также локально антиподальные множества. Теорема 4 утверждает, что этим исчерпываются все локально антиподальные множества.

Теорема 4 (о структуре локально антиподальных множеств). Всякое локально антиподальное множество Делоне $X \subset \mathbb{R}^{d}$ представимо в виде

$$
X=\bigcup_{i=1}^{N}\left(x+v_{i}+\Lambda\right),
$$

где $x \in \mathbb{R}^{d}$ - некоторая точка, $\Lambda$ - $d$-мерная решетка, а векторы $v_{1}, v_{2}, \ldots, v_{N}(1 \leqslant$ $\left.N \leqslant 2^{d}-1\right)$ - представители некоторых смежсных классов из факторрешетки $\frac{\Lambda}{2} / \Lambda$.

Таким образом, локально антиподальное множество Делоне является кристаллом, состоящим не более чем из $2^{d}-1$ решеток, которые попарно конгруэнтны, параллельно ориентированы и специальным образом сдвинуты (на векторы с полуцелыми координатами).

\section{Список литературы}

[1] Б. Н. Делоне, УМН, 1937, № 3, 16-62. [2] Б.Н.Делоне, Н. П. Долбилин, М. И. Штогрин, Р. В. Галиулин, Докл. АН СССР, 227:1 (1976), 19-21. [3] Н. П. Долбилин, Вестник ЧелГУ, 2015, № 3(358), 6-17.

Н. П. Долбилин (N. P. Dolbilin)

Математический институт им. В. А. Стеклова

Российской академии наук

E-mail: dolbilin@mi.ras.ru
Представлено В. М. Бухштабером Принято редколлегией 03.09.2015

\section{А. Н. Магазинов (А. N. Magazinov)}

Математический институт им. В. А. Стеклова

Российской академии наук

E-mail: magazinov-al@yandex.ru 